\title{
The Present Status of Maintenance Strategies and the Impact of Maintenance on Reliability
}

\author{
A Report of the IEEE/PES Task Force on Impact of Maintenance Strategy on Reliability of the Reliability, \\ Risk and Probability Applications Subcommittee, J. Endrenyi, S. Aboresheid, R. N. Allan, G. J. Anders, \\ S. Asgarpoor, R. Billinton, N. Chowdhury, E. N. Dialynas, M. Fipper, R. H. Fletcher, C. Grigg, J. McCalley, \\ S. Meliopoulos, T. C. Mielnik, P. Nitu, N. Rau, N. D. Reppen, L. Salvaderi, A. Schneider, and Ch. Singh
}

\begin{abstract}
In this paper, the most frequently used maintenance strategies are reviewed. Distinction is made between strategies where maintenance consists of replacement by a new (or "good as new") component and where it is represented by a less costly activity resulting in a limited improvement of the component's condition. Methods are also divided into categories where maintenance is performed at fixed intervals and where it is carried out as needed. A further distinction is made between heuristic methods and those based on mathematical models; the models themselves can be deterministic or probabilistic.

From a review of present maintenance policies in electric utilities it is concluded that maintenance at fixed intervals is the most frequently used approach, often augmented by additional corrections. Newer "as needed"-type methods, such as reliability-centered maintenance (RCM), are increasingly considered for application in North America, but methods based on mathematical models are hardly ever used or even considered. Yet only mathematical approaches where component deterioration and condition improvement by maintenance are quantitatively linked can determine the effect of maintenance on reliability. Although more complex, probabilistic models have advantages over deterministic ones: they are capable of describing actual processes more realistically, and also facilitate optimization for maximal reliability or minimal costs.
\end{abstract}

Index Terms-Maintenance, overhaul, power system reliability, probability: applications, reliability modeling.

\section{INTRODUCTION}

I N 1995, a Task Force was established by the IEEE Subcommittee on Application of Probability Methods (presently Reliability, Risk \& Probability Applications SC) to investigate maintenance strategies and their effect on reliability. The TF concluded its work in 1999 and the present paper is a condensation of its full report [21].

The purpose of maintenance is to extend equipment lifetime, or at least the mean time to the next failure whose repair may be costly. Furthermore, it is expected that effective maintenance policies can reduce the frequency of service interruptions and the many undesirable consequences of such interruptions. Maintenance clearly affects component and system reliability: if too little is done, this may result in an excessive number of costly failures and poor system performance and, therefore, reliability

Manuscript received December 20, 1999; revised December 13, 2000.

Task Force Chair: J. Endrenyi, Ontario Power Technologies. Members listed above.

Publisher Item Identifier S 0885-8950(01)09455-X is degraded; done too often, reliability may improve but the cost of maintenance will sharply increase. In a cost-effective scheme, the two expenditures must be balanced.

Maintenance is just one of the tools for ensuring satisfactory component and system reliability. Others include increasing system capacity, reinforcing redundancy and employing more reliable components. At a time, however, when these approaches are heavily constrained, electric utilities are forced to get the most out of the devices they already own through more effective operating policies, including improved maintenance programs. In fact, maintenance is becoming an important part of what is often called asset management.

Electric utilities have always relied on maintenance programs to keep their equipment in good working condition for as long as it is feasible. In the past, maintenance routines consisted mostly of pre-defined activities carried out at regular intervals (scheduled maintenance). However, such a maintenance policy may be quite inefficient: it may be too costly (in the long run), and may not extend component lifetime as much as possible. In the last ten years, many utilities replaced their fixed-interval maintenance schedules with more flexible programs based on an analysis of needs and priorities, or on a study of information obtained through periodic or continuous condition monitoring (predictive maintenance).

The predictive maintenance routines include a group of programs named Reliability-Centered Maintenance, commonly abbreviated to RCM. In an RCM approach, various alternative maintenance policies can be compared and the one most cost-effective for sustaining equipment reliability selected. RCM programs have been installed by several electric utilities as a useful management tool.

The implementation of RCM programs represented a significant step in the direction of "getting the most out" of the equipment installed. However, the approach is still heuristic, and its application requires experience and judgment at every turn. Besides, it can take a long time before enough data are collected for making such judgments. For this reason, several mathematical models have been proposed to aid maintenance scheduling. In fact, the literature on maintenance models has become quite extensive.

In the following, first a short review is given of the most important approaches and models described in the literature. Next, present maintenance policies are examined using the results of a survey initiated by the Task Force and involving electrical utilities in several countries. Subsequently, the use of mathematical 


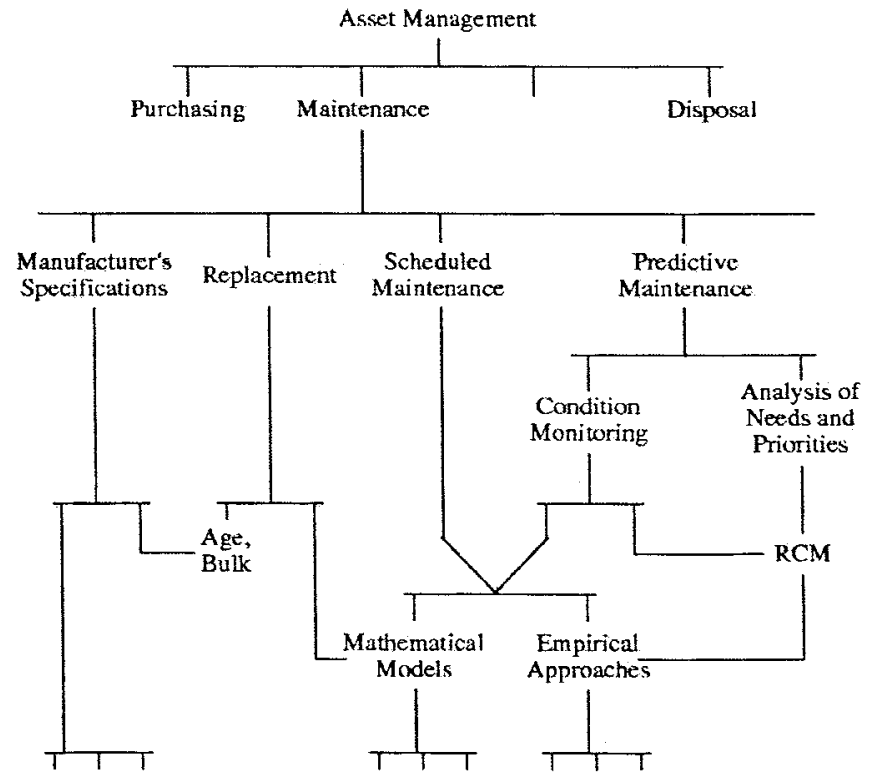

Fig. 1. Overview of maintenance approaches.

models for maintenance strategies is explored and desirable attributes of realistic probability-based models are listed. In the closing section, reference is made to the role of maintenance in deregulated power system operation. In Appendix I, definitions are given of the most important concepts discussed in the paper.

\section{REVIEW OF MAINTENANCE APPROACHES}

A classification of the various maintenance approaches is presented in Fig. 1. Note that maintenance is shown as part of the overall asset management effort. Clearly, maintenance policy is one of the operating policies and, in a given setting, it is selected to satisfy both technical requirements and financial constraints.

Much of the literature concerns itself with replacements only, both after failures and during maintenance, and disregards the possibility of the kind of maintenance where less improvement is achieved at smaller cost. The oldest replacement schemes are the age replacement and bulk replacement policies [1], [2]. In the first, a component is replaced at a certain age or when it fails, whichever comes first. In the second, all devices in a given class are replaced at predetermined intervals, or when they fail. The last policy is easier to administer (especially if the ages of components are not known) and may be more economical than a policy based on individual replacement. Newer replacement schemes are often based on probabilistic models (e.g., [3], [4]) and can be quite complex. In most electrical utility applications, however, maintenance resulting in limited improvement is an established practice and replacement models have only a secondary role.

Maintenance programs range from the very simple to the quite sophisticated. Perhaps the simplest plan is to adopt a rigid maintenance schedule where pre-defined activities are carried out at fixed time intervals. Whenever the component fails, it is repaired or replaced. Both repair and replacement are assumed to be much more costly than a single maintenance job. The maintenance intervals are selected on the basis of long-time experience (not necessarily an inferior alternative to mathematical models). To this day, this is the approach most frequently used.

The RCM approach referred to in the Introduction is heavily based on regular assessments of equipment condition and, therefore, does not apply rigid maintenance schedules. It should be observed that RCM is a somewhat fluid concept, defined differently in various sources [11]-[13]. It is not always based on condition monitoring, but on other features such as failure modes and effects analysis and an investigation of operating needs and priorities. The approach is almost always empirical. As an example, the RCM program used at the Consolidated Edison Company of New York [11] consists of the following procedure.

- System identification, and the listing of critical components and their functions.

- Failure mode and effects analysis for each selected component, the determination of failure history, and the calculation of mean time between failures.

- Categorization of failure effects (by using appropriate flow charts) and determination of possible maintenance tasks.

- Maintenance task assignment.

- Program evaluation, including cost analysis.

Another approach, claimed to be more efficient than RCM, was recently described in Reference [14]. Called Preventive Maintenance Optimization (PREMO), it is based on extensive task analysis rather than system analysis, with a capability of drastically reducing the required number of maintenance tasks in a plant. Programs such as RCM and PREMO have been very useful in ensuring the economic operation of power stations. However, they will not provide the full benefits and flexibility of programs based on mathematical models.

For a complete evaluation of the effects of a maintenance policy, one had to know by how much its application would extend the lifetime of a component, measured in, say, mean time to failure. To find this out, a mathematical model of the component deterioration process is required, which is then combined with a model describing the effects of maintenance. In the last ten years or so, several such models have been proposed [5]-[7]. They provide the link missing in earlier approaches: a quantitative connection between reliability and maintenance. Once a mathematical model is constructed, the process can be optimized with regard to changes in one or more of the variables.

The simpler mathematical models are essentially still based on fixed maintenance intervals, and optimization will result in identifying the least costly maintenance frequency. More complex models incorporate the idea of condition monitoring, where decisions with regard to the timing and amount of maintenance are dependent on the actual condition (stage of deterioration) of the device [5], [8], [9]. Thus, some kind of monitoring (e.g., inspection) must be part of the model [10]. Other desirable features are listed in Section VI.

Mathematical models may be deterministic or probabilistic. A discussion of the two approaches is given in Section V. Probabilistic models developed for applications in the electric power industry are described in References [17]-[20]. Good surveys of the literature on maintenance are given in References [15] and [16]. 
TABLE I

REPORTED Most FREQUENT MAINTENANCE INTERVALS AND DURATIONS

\begin{tabular}{|c|c|c|c|c|c|c|}
\hline & \multicolumn{2}{|c|}{ A: Generators } & \multicolumn{2}{|c|}{ B: Transformers } & \multicolumn{2}{|c|}{ C: Breakers } \\
\hline & Interval & Duration & Interval & Duration & Interval & Duration \\
\hline Minor maintenance & $1 \mathrm{yr}$ & $1-2 \mathrm{wk}$ & $1 \mathrm{yr}$ & 1 day & $1 \mathrm{yr}$ & 1 day \\
\hline Minor overhaul & $5 \mathrm{yr}$ & $4-5 w k$ & $5 \mathrm{yr}$ & 3 days & $5 \mathrm{yr}$ & 3 days \\
\hline Major overhaul & $8-10 y r$ & $6-8 \mathrm{wk}$ & $7 \mathrm{yr}$ & $4-8 \mathrm{wk}$ & $8-10 \mathrm{yr}$ & $2 \mathrm{wk}$ \\
\hline
\end{tabular}

\section{Present Maintenance Policies in Electric Utilities}

To form an overview of present maintenance practices, a questionnaire was prepared and, with the help of the Task Force members, distributed among a number of utilities, both in North America and overseas. Since maintenance protocols vary from equipment to equipment and to review all would have required an unwieldy effort, it was decided to select three typical components of different sizes and quantities in the system as representative in the hope that basic trends can already be observed on this sample. The equipment selected are: A) hydrogen-cooled steam-turbine driven generators, B) substation transformers, $100-161 \mathrm{kV}$ primary, 4-20 kV secondary, and C) distribution system indoor circuit breakers, $15 \mathrm{kV}$. Accordingly, the questionnaire was structured into three parts.

In each part, questions were asked about the maintenance policy presently in effect and the criteria for triggering the next maintenance activity; about using, or planning to use, such advanced strategies as RCM or the ones based on mathematical models; and about the available data-base. A sample questionnaire (Part B) is shown in Appendix II.

Replies were received from 6 countries, Austria, Canada, Germany, Italy, Saudi Arabia, and the U.S. A total of 53 completed questionnaires were returned, 19 of Part A, and 17 each of Parts B and C. Thus the returns form comparatively small samples, but even so, the conclusions can be stated with some measure of confidence. In the following, the findings are listed.

\section{A. General}

- The answers to many questions display a considerable spread. This is not only the consequence of different practices, but also of different interpretations of some of the concepts introduced.

- Many utilities do scheduled maintenance only (11 out of 53 ), or a modified form of it where additional corrective actions are taken if required by inspection results (36). Only in a few cases (6) was exclusive use of predictive maintenance reported.

\section{B. Scheduled Maintenance}

- The intervals and durations reported for scheduled maintenance show considerable spread. Table I lists their most frequent values.

- Cyclic routine (e.g., a major overhaul following 3 minor overhauls) is rare (6 cases reported).

\section{Predictive (as Needed) Maintenance}

- The most often used device to establish the need for maintenance is periodic inspection. The inspection intervals vary widely and are also different for different tasks. For example, in Part B intervals ranging from 1 week to 5 years were reported, with the most frequent entry of 1 month. Same for Part C. In Part A, yearly inspections were the most frequent.

- Another device for detecting maintenance needs is continuous monitoring. This was mentioned most often in Part A (oil leakage, vibration, bearing temperature) and to lesser degree for smaller equipment (tap changer condition, corrosion, discharge voltage).

- The most effective diagnostic tools were found to be, in Part A, gas and oil analysis, surge testing, vibration monitoring; in Part B, gas and oil analysis, power factor tests, thermal tests, dielectric tests; in Part C, contact resistance tests, hi-pot tests.

\section{Reliability-Centered Maintenance (RCM)}

- This procedure is not generally used, and particularly rarely applied outside North America. However, nearly half of the correspondents are considering its introduction. At the present, only 1 in Part A, 2 in Part B and 1 in Part $\mathrm{C}$ reported that the procedure is fully used.

- Those who consider using RCM are expecting to gain the following benefits: longer up-times, lower costs, better control and decisions, better use of labor.

\section{E. Probabilistic Models}

- Probabilistic approaches are not used in maintenance planning by any of the respondents. Some report on pilot applications and "tests," others have hired external consultants who may include such methods.

- Many do, or wish to, compute such indices as unavailability, failure frequency and duration (or mean time to failure); in Part A also forced outage rate (FOR) or equivalents.

\section{F. Data Requirements}

- For generators (Part A), present maintenance policies are primarily based on historical records. These may include performance indices, inspection records and maintenance data. In addition, generator manuals are used and experience and memory were frequently mentioned as important resources. 


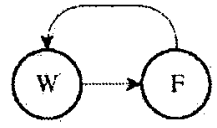

(a)

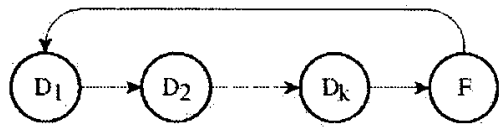

(b)
Fig. 2. State diagrams for (a) random failure and (b) deterioration failure W: working state, F: failure state, $D_{1}, D_{2}, \ldots$ : stages of deterioration.

- For transformers (Part B), the more frequently mentioned data used were test reports, data on windings, failure data, maintenance protocols and maintenance history.

- For breakers (Part C), the replies included operation logs, maintenance history, failure statistics, faulty operation counts vs. total number of operations, results of oil and hi-pot tests.

\section{G. Contracting Out Maintenance Work}

- The majority of respondents do contract out at least part of the maintenance activities. Some do it on an "as needed" basis or only for special tests. Others contract out major maintenance work.

\section{Mathematical Models Linking ReliabiLity AND MAINTENANCE}

\section{A. The Use of Mathematical Models}

As mentioned in Section II, the simplest maintenance policies consist of a set of instructions taken from equipment manuals or based on long-standing experience. There are no quantitative relationships involved and the possibilities are very limited for making predictions about the effectiveness of the policy or carrying out any sort of optimization. To make numerical predictions and carry out optimizations, mathematical models are needed which can represent the effects of maintenance on reliability.

Mathematical models can be deterministic or probabilistic. Both can be put to good use in appropriate maintenance studies, and many applications are described in the literature [10]. However, where quantities are involved whose values vary randomly, the associated future uncertainties can be properly handled only through probabilistic models. In the following, a probabilistic approach to linking maintenance and reliability is described.

\section{B. Failure Models}

In many applications, component failures can be divided into two categories, random failures and those arising as a consequence of deterioration (aging). Simple failure-repair processes in the two cases are shown in Fig. 2. The various state designations are explained in the legend of the figure.

The deterioration process is represented by a sequence of stages of increasing wear, finally leading to equipment failure. Deterioration is of course a continuous process in time and only for the purposes of easier modeling is it considered in discrete steps. The number of deterioration stages may vary, and so do their definitions. There are, essentially, two ways of defining deterioration stages: either by duration (the second stage is reached, on the average, in three years, the third in six, and so on), or by physical signs (corrosion, wear, etc.) and appropriate

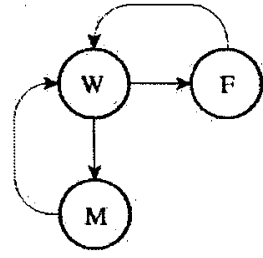

(a)

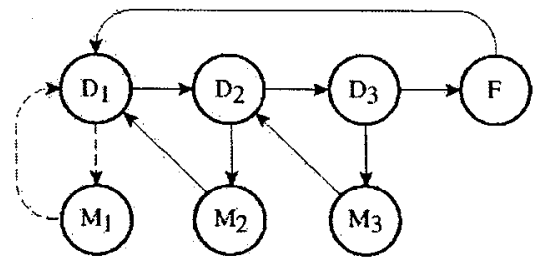

(b)
Fig. 3. State diagrams including maintenance states for (a) random failure, (b) failure following a three-stage deterioration process.

mileposts. In practical applications, the tendency is to favor the second way which, of course, makes periodic inspections necessary to determine the stage of deterioration the device has reached. If this course is taken, the mean times between the stages are usually uneven, and are selected from performance data or by judgment based on experience.

The processes in Fig. 2 can be readily represented by probabilistic mathematical models. If the transitions shown between the states are assumed to occur at constant rates, the mathematical models describing these processes are known as Markov models. Well-known techniques exist for the solution of such models.

The assumption of a constant failure rate explains why the model in Fig. 2(a) represents random failure: since the rate of failure is constant, the chance of a failure occurring in any future $\Delta t$ time-interval is the same (see Appendix I, definition of Random failure). Note that a constant failure rate (or hazard rate) model is a special case where the hazard function, associated with the probability of failure in a future $\Delta t$ time interval if the device is still working at the beginning of $\Delta t$, is constant. In most practical cases the hazard function increases with time (see Appendix I, Note 1 to entry Random failure).

It can be proven that in a Markov model the times of stay in the various states are exponentially distributed. This property and the constant-rate property follow from each other. The mean time to failure from the instant a "new" device is put into service - that is, the moment of entrance into the $\mathrm{W}$ state in case (a) and into the initial deterioration state $\mathrm{D}_{1}$ in case (b) - is the mean time spent in $\mathrm{W}$ in the first case, and the sum of the mean durations of the deterioration states in the second. It is important to realize that this sum represents a time-variable which is not exponentially distributed.

\section{The Effect of Maintenance}

As already mentioned, the purpose of maintenance is to increase the mean time to failure. One way of adding maintenance states to the models in 0.2 is shown in Fig. 3. In diagram 3(b), it is assumed that maintenance will bring about an improvement to the conditions in the previous stage of deterioration (minimal maintenance, [6]). This contrasts with many strategies described in the literature, where maintenance involves replacement - that is, a return to the "new" conditions.

In the case of random failures [Fig. 3(a)], the constant failure-rate assumption leads to the result that maintenance cannot produce any improvement, because the chances of a failure occurring during any future time-interval are the same with or without maintenance. This finding also agrees with 
experience; it gave rise to the widely known piece of wisdom: "if it ain't broke, don't fix it!" The situation is quite different for deterioration processes, where the times from the new condition to failure are not exponentially distributed even if the times between subsequent stages of deterioration are. In such a process the hazard function is increasing, and maintenance will bring about improvement independently of the types of distributions between stages [7]. Hence the rule: conditions cannot be improved by maintenance for random failures, but maintenance has an important role to play when failures are the consequence of aging.

In Fig. 3(b), the dotted-line transitions to and from state $\mathrm{M}_{1}$ indicate that maintenance out of $\mathrm{D}_{1}$ should really not be performed because, as noted, it would be meaningless. State $\mathbf{M}_{1}$ could be indeed omitted if the maintainer knew that at the time of next maintenance the deterioration process would still be in its first stage and, therefore, no maintenance would be needed. Otherwise, maintenance must be carried out regularly from the beginning, and state $\mathrm{M}_{1}$ has to be part of the diagram.

Note that deterioration processes are a subset of the failure models where the hazard function is increasing with time. Maintenance is advantageous in all such cases. If the failure is random, the hazard function is constant, and maintenance is of no use.

As already mentioned, the identification of the deterioration stage a device finds itself in at any given time is a significant part of the methodology and models. The next section deals with approaches to this task.

\section{Identification of the Deterioration Stages}

Under a predictive maintenance policy, maintenance is carried out as needed. There are no schedules to follow. The need for maintenance is established through periodic or continuous inspection.

To perform meaningful periodic inspections, diagnostic routines and techniques are required which help to identify disorders that call for maintenance. While the maintenance activities are performed as needed, inspections should be carried out regularly to initiate maintenance before equipment break-down. In addition, predictive maintenance may allow for:

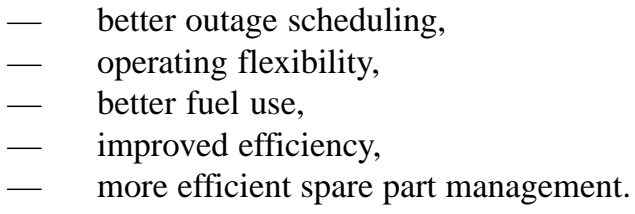

Commonly used diagnostic methods include visual inspection, optical inspection, neutron analysis, radiography, eddy current testing, ultrasonic testing, vibration analysis, lubricant analysis, temperature analysis, magnetic flux leakage analysis and acoustic emission monitoring. Each of these methods has advantages and limitations.

Continuous inspection, or condition monitoring, is the ongoing inspection and surveillance of the operation of equipment to ensure proper performance and to detect abnormalities indicative of approaching failure. Condition monitoring is preferred where it is not possible to predict wear-out trends through periodic inspections with reasonable accuracy, given that the
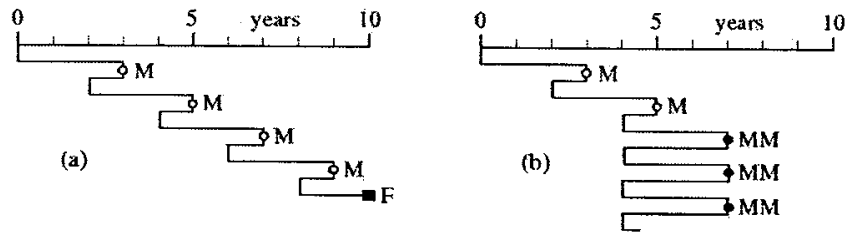

Fig. 4. Maintenance every 3 years, resulting in (a) 1-year improvement, (b) 3-year improvement if total wear is 6 years or more, otherwise as in (a). $\mathrm{M}$-maintenance $\mathrm{MM}$-overhaul $\mathrm{F}$-failure.

associated costs are not prohibitive; also, where off-line inspections are not desirable and where the criticality of a failure justifies keeping constant vigil on a device or process. In fact, if the costs are not excessive, condition monitoring may be more economical than maintenance based on regular inspections [18].

\section{E. Other Models}

The model described in Section IV-C represents just one of several ways of accounting for the effect of maintenance on reliability. It is the basis of the program described in [18]. Many other approaches have been developed; of these, at least two are concerned with power system applications [19], [20]. In [19], a maintenance model is derived for parallel branches of components in series as often found in transformer stations, and in [20], the maintenance and reliability of standby devices are studied. Both are, in essence, replacement models where repair and maintenance are assumed to result in "as new" conditions.

\section{DETERMINISTIC OR PROBABILISTIC APPROACH?}

As mentioned before, maintenance can increase the time to failure only if failures do not happen at random but are the consequences of deterioration occurring as a device ages. Therefore, any mathematical model to represent the benefits of a given maintenance policy must relate the results of maintenance to the process of deterioration. A simple probabilistic model where such a link is established was shown in Fig. 3(b); its properties were discussed in Section IV-C. A prominent feature of the model is that its solution can be readily optimized either for the highest reliability or for the lowest cost.

While many deterministic maintenance models have been proposed, a simple one could be devised by using roughly the same approach as that used in the development of the probabilistic model earlier. An example is shown in Fig. 4(a), based on the assumptions that without maintenance, the device would fail after exactly 10 years, the (rigid) maintenance interval is 3 years and the effect of maintenance is a 1-year improvement in deterioration (a questionable assumption; more of which later). The horizontal line serves as a scale of deterioration; otherwise the diagram in Fig. 4(a) is self-explanatory. Deterioration and maintenance are still linked through an algorithm based on the diagram; this algorithm takes the place of a mathematical model. It can be seen that the time to failure is now extended to 14 years as a result of the four maintenances carried out in the interval.

While it is conceivable that the improvement due to a maintenance activity is less than the deterioration between two consecutive maintenances, especially early in the life of a device 
when only minor maintenances are performed, later the effect of maintenance should equal or exceed the deterioration occurring between maintenances. This can be ensured by scheduling overhauls (major maintenances) beyond a given stage of deterioration. If, for instance, in the example of Fig. 4(a) overhaul is required at or after the deterioration stage 6 , and if the effect of overhaul is a 3 -year improvement in deterioration, the diagram will change to that shown in Fig. 4(b). Note that now the expected time to failure is infinity.

The problem with this deterministic representation (and many others) becomes obvious in the last example. It is easy to visualize that if the setback effected by maintenance is less than the maintenance interval the process will tend "to the right" and end in failure. However, this can be considered an unlikely case. Every time the setback equals the maintenance interval, the process will oscillate within a given range, as in Fig. 4(b), and if it exceeds the maintenance interval, the process will move "to the left." In both latter cases the implication is that failure will never occur. This is a false conclusion and is entirely due to the assumption that all quantities involved have fixed values. If variability is allowed as in a probabilistic model, the failure state will sooner or later always be reached. This agrees with experience and can be proven rigorously.

In conclusion, it appears that while deterministic approaches may be simple to follow, some can lead to erroneous results. Probabilistic models produce much more credible conclusions but, unfortunately, their more complex structure will often mask the fact that they can in many applications better describe real conditions.

\section{MODEL EXTENSIONS}

In general, probabilistic models can accommodate extensions and refinements easier than deterministic models. In the following, a few examples are given of desirable features that ought to be built into maintenance models.

If the rates of transitions from the $\mathrm{D}_{i}$ to the $\mathrm{M}_{i}$ states are the same for all $i$, the model in Fig. 3(b) will represent a situation where maintenance is scheduled; that is, performed at regular intervals. It is fairly obvious that better economy can be achieved with a predictive maintenance policy where maintenance is performed only when needed. The model in Fig. 3(b) can be further developed to accommodate such a policy [18]. A simple solution is to insert inspection states before proceeding to maintenance; during inspections decisions are made as to the necessity of carrying out maintenance at that time. The probabilities of the possible decision outcomes must be chosen in advance; clearly, the more elaborate is the model, the more input data are needed.

Further development is required for enhancing probabilistic models so that they can accommodate, for example, the following:

- maintenance schemes based on continuous condition monitoring instead of periodic inspection;

- several deterioration processes taking place concurrently;

- the impact of load changes, or cycling loads, on aging;

- the possibility that maintenance, if not done with care, can damage rather than improve the condition of a device; in fact, the effect of maintenance in terms of the resulting adjustment in the deterioration process should be considered a random variable, including the possibilities of no set-back and "set-forward";

- recognition of the necessity of postponing maintenance, particularly for generating units, if load conditions do not allow the removal of a unit from service;

- Recognition of the effect of obsolescence which may result in spare part unavailability at some time during the device's active lifetime.

The claim may be made that the effect of the break-in/ debugging period at the beginning of a device's operating life, when failure rate is possibly quite high, should also be recognized in a practical model. However, maintenance during this period is usually covered by special instructions which are apart from the long-term maintenance policy, the subject matter of this report.

Many of the above refinements are presently under study. Probabilistic models dealing with some of these concerns are already described in the literature (e.g., [10], [18]-[20]). It needs to be emphasized that the present effort is not aimed at developing a comprehensive maintenance program. The goal is to increase the industry's awareness of this tool for improving reliable operation, a tool whose use has not been fully exploited in the past.

\section{OUTLOOK}

From the limited survey of maintenance practices in electric utilities described in this report it has become evident that many utilities in North America and overseas have not yet adopted practices beyond those of scheduled maintenance or empirical forms of predictive maintenance based on periodic inspections. Mathematical models, deterministic or probabilistic, are as of yet rarely used. They lack the simplicity required for evaluations which are often carried out in the field; besides, they require a multitude of input information which may not be readily available.

For all their advantages, probability-based maintenance policies are particularly slow in being considered for implementation. It cannot be denied, however, that optimized probabilistic maintenance models would provide the highest savings and also the highest flexibility in exploring and utilizing the effects of changes in any of the parameters. Therefore, there is little doubt that the final development in utility maintenance policies will be the introduction of such models, however complex they first appear to be. This is particularly true in a competitive environment where it is a prime necessity to find optimal solutions in complicated situations. A good example is generator maintenance where, as mentioned above, the questions are not only of minimizing maintenance and repair costs but also of appropriate scheduling.

In the past, the practice of electric utilities and power pools was to centrally plan and coordinate the maintenance of generating units. Maintenance was done during low-load seasons and the timing was influenced by such considerations as system risk and production cost. In the deregulated scenario, maintenance may not be centrally planned or even coordinated. Generator owners may tend to keep the units running when the market 
clearing price of electric energy is high and perform maintenance only when the market price is low. In addition, they may wish to sell energy in a neighboring control area in which the periods of high load (and high market price) may be different from those in the area where the generator is located. Under such circumstances the decision when to maintain a generator will be driven by profit incentives rather than by the optimal cost of maintenance and repair.

Since it is unclear at the time of this writing how energy markets will operate, it is not possible to examine the effect of generator maintenance policies on risk in the deregulated industry. Therefore this report does not address these issues. But it should be noted that for equipment other than generating units and some generating station components, findings in this document are applicable even when utilities operate in competition. Work is being carried out in several centers to develop program packages for the probability-based maintenance approaches discussed in this report, for risk analysis under deregulation and how risk is influenced by various maintenance strategies.

\section{APPENDIX I \\ TERMINOLOGY}

In the following, definitions are given of a few fundamental concepts discussed in this paper. It must be understood that no standard nomenclature exists in this field; in the notes to the definitions, several alternative terms are listed which are frequently used in power system reliability studies or in the component and circuit reliability literature. In proposing the definitions below, an attempt is made to offer a consistent set which may be acceptable to most users.

Failure: The termination of the ability of a device to perform a required function.

Random Failure: A failure whose rate of occurrence (intensity) is constant, and independent of the device's condition.

Note 1: The chances of a random failure occurring in any short time interval, assuming that the device has been working up to that time, is always the same. In the more precise terminology of reliability theory, a failure is random if the density of the conditional probability that it occurs in the interval $(t, t+\Delta t)$, given that the device was in a working condition at $t$, is constant (independent of $t$ ). In general, this density is called the hazard function, and if it is constant, the hazard rate or failure rate.

Note 2: In a broader sense, failures whose origins are not well understood and therefore are perceived as being able to occur at any time are often said to be random. If for easier mathematical modeling it is assumed that such failures can occur at any time with equal probability, then the broader concept is reduced to the above definition.

Note 3: The rate of random failure may depend on external conditions. For example, the rates of lightning or ice storms, and the rates of resulting random failures, would be different in each season.

Deterioration (Wear or Wear-Out): A process by which the rate of failure increases due to loss of strength, the effects of usage, environmental exposure or passage of time.
Note: The term is also used to describe the accumulated results of the process.

Deterioration Failure: A failure resulting from the deterioration of a device.

Restoration: An activity which improves the condition of a device. If the device is in a failed condition, the intent of restoration is the re-establishment of a working state.

Replacement: Restoration wherein a device is removed and one in better condition is put in its place; if the device is failed, it is replaced by a working one. It is often assumed that the device so installed is new.

Repair: Restoration wherein a failed device is returned to operable condition.

Note: It is common to use the term corrective maintenance for both replacement and repair.

Minimal Repair: Repair of limited effort wherein the device is returned to the operable state it was in just before failure.

Maintenance: Restoration wherein an unfailed device has, from time to time, its deterioration arrested, reduced or eliminated.

Note: It is common to call this concept planned maintenance or preventive maintenance. These terms are meant to contrast with corrective maintenance (see Repair). They are replaced by the above definitions and not used in this paper.

Scheduled Maintenance: A maintenance carried out at regular intervals (rigid schedule).

Note: Another term often used for this activity is preventive maintenance. This usage of the term contradicts the one mentioned in the Note to Maintenance above. In this report, the term is not used.

Predictive Maintenance: A maintenance carried out when it is deemed necessary, based on periodic inspections, diagnostic tests or other means of condition monitoring.

Emergency Maintenance: A predictive maintenance that must be carried out immediately, or with the shortest delay possible, after condition monitoring detects a danger of imminent failure.

Minor Maintenance: Maintenance of limited effort and effect.

Note: If deterioration is modeled in discrete stages and the intent of maintenance is to improve conditions by just one stage, the maintenance procedure is often called minimal.

Overhaul: Maintenance or repair requiring major effort and resulting in a significant improvement of the device's condition.

Note: Occasionally the terms maintenance-overhaul and repair-overhaul are used to indicate the distinction. In most cases, however, this is not necessary and these terms will not be used in this report.

Minor Overhaul: An overhaul of substantial effort yet involving only a limited number of parts, whose effect is a considerable improvement of the equipment's condition.

Major Overhaul: An overhaul of extensive effort and duration which involves most or all parts of the equipment and results, as far as possible, in the "good as new" condition. 
18) Do you contract out any maintenance work (e.g., major overhaul)?

If yes, what type and what percentage? $\%$

\section{REFERENCES}

[1] R. E. Barlow and F. Proschan, Statistical Theory of Reliability and Life Testing. New York: Holt, Rinehart and Winston, Inc., 1975.

[2] G. J. Anders, Probability Concepts in Electric Power Systems. New York: J. Wiley \& Sons, 1990.

[3] X. Zheng and N. Fard, "A maintenance policy for repairable systems based on opportunistic failure-rate tolerance," IEEE Trans. Reliability, vol. 40, no. 2, pp. 237-244, June 1991.

[4] N. Ebrahimi, "Two new replacement policies," IEEE Trans. Reliability, vol. 42, no. 1, pp. 141-147, Mar. 1993.

[5] R. V. Canfield, "Cost optimization of periodic preventive maintenance," IEEE Trans. Reliability, vol. 35, no. 1, pp. 78-81, April 1986.

[6] S. H. Sim and J. Endrenyi, "Optimal preventive maintenance with repair," IEEE Trans. Reliability, vol. 37, no. 1, pp. 92-96, Apr. 1988.

[7] J. Endrenyi and S. H. Sim, "Availability optimization for continuously operating equipment with maintenance and repair," in Proceedings of the Second PMAPS Symposium, September 1988, Nov. 1989, EPRI Publication EL-6555.

[8] G. J. Anders et al., "Maintenance planning based on probabilistic modeling of aging in rotating machines," in CIGRE Conference, Paris, 1992, Paper no. 11-309.
[9] B. Reichman et al., "Application of a maintenance planning model for rotating machines," in CIGRE Conference, Paris, 1994, Paper no. 11-204.

[10] A. K. S. Jardine, Maintenance, Replacement and Reliability. London: Pitman Publishing, 1973.

[11] Reliability Centered Maintenance Seminar. New York: A publication of the Consolidated Edison Company, 1988.

[12] A. M. Smith, Reliability-Centered Maintenance. New York: McGrawHill, Inc., 1993.

[13] J. Moubray, Reliability-Centered Maintenance. New York: Industrial Press Inc., 1992.

[14] Wisconsin Public Service Corp., "PM optimization boosts reliability at small cost," Utility Best Practices, p. 157, Oct. 1995.

[15] H. Ascher and H. Feingold, Repairable Systems Reliability. New York: Marcel Dekker, Inc., 1984.

[16] C. Valdez-Flores and R. M. Feldman, "A survey of preventive maintenance models for stochastically deteriorating single-unit systems," Naval Research Logistics Quarterly, vol. 36, pp. 419-446, Aug. 1989.

[17] EPRI, "Probabilistic model to estimate the remaining life of generator station components," Report RP-2577-1, 1993.

[18] J. Endrenyi, G. J. Anders, and A. M. Leite da Silva, "Probabilistic evaluation of the effect of maintenance on reliability-An application," IEEE Trans. Power Systems, vol. 13, no. 2, pp. 576-583, May 1998.

[19] R. Billinton and J. Pan, "Optimal maintenance scheduling in a parallel redundant system of series components in each branch," IEEE Trans. Power Systems, to be published.

[20] E. N. Dialynas and D. G. Michos, "Time-dependent unavailability analysis of standby components incorporating arbitrary failure distributions and three inspection/maintenance policies," Reliability Engineering and System Safety, vol. 39, pp. 35-54, 1993.

[21] IEEE/PES Task Force on Impact of Maintenance Strategy on Reliability, "Impact of maintenance strategy on reliability," Final Report, July 1999. 